\title{
Learning Opportunities for Sharing Data in the ARIADNE Project
}

\author{
Franco Niccolucci \\ PIN, VAST-LAB \\ 25 Piazza G. Ciardi, Prato, Italy \\ franco.niccolucci@gmail.com
}

\author{
Paola Ronzino \\ PIN, VAST-LAB \\ 25 Piazza G. Ciardi, Prato, Italy \\ p.ronzino@gmail.com
}

\section{INTRODUCTION}

Advanced Research Infrastructure for Archaeological Dataset Networking (ARIADNE) is a four-year EU FP7 Infrastructures funded project, made up of 24 partners across 16 European countries. ARIADNE has the goal of "bringing together and integrating existing archaeological research data infrastructures, so researchers can use the various distributed datasets and new and powerful technologies as an integral component of the archaeological research methodology". A fundamental component in meeting this goal is the provision of transnational access (TNA) to a wide variety of people involved in visualising data. To accomplish this, online access opportunities are being developed, but funding will also be provided for on-site training with our technical partners. This will be a rare opportunity to work directly with the staff designing the infrastructure, including PINUniversity of Florence at Prato, Consiglio Nazionale delle Ricerche (CNR) in Pisa, Athena RC - Digital Curation Unit (DCU) in Maroussi, and Athena RC Cultural and Technology Institute (CETI) in Xanthi. Technical staff will be on hand to present information about the training (including logistical details and application procedures), to discuss the goals of the project, and answer questions.

\section{ARIADNE PHYSICAL TNA - SUMMER SCHOOLS}

ARIADNE is offering three summer schools, for the year 2014, addressing researchers that will benefit from training in archaeological research data management to carry forward their own research. Individual work, indeed, will form part of the summer school programme through case studies or research projects, which will be proposed by participants in advance and developed by them under the experts' supervision.

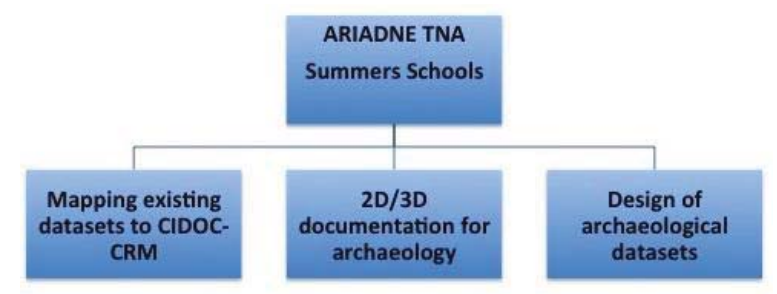

Figure 1: ARIADNE summer schools for 2014

\subsection{Mapping existing datasets to CIDOC CRM}

The "Mapping existing databases to CIDOC CRM" summer school is organized by VAST-LAB of PIN, the Prato branch of the University of Florence. Since 2001 VAST-LAB has developed significant expertise in the field of archaeological applications and has advised institutions in Italy and abroad on the recovery of legacy data and conversion to updated data formats, inclusion in data portals, and the creation of archaeological datasets.

The summer school aims at enabling researchers and professionals to map their datasets to the CIDOC CRM standard. This process is necessary to integrate them in a wider framework such as ARIADNE. The school will provide a summary background of CIDOC CRM showing some case studies and some frequently used templates. The final days will be dedicated to developing the mappings of students' case studies, which they will carry out under the supervision of the specialists.

\subsection{D/3D documentation for archaeology}

The Visual Computing Lab (VCLab), one of the research departments of the Istituto di Scienza e Tecnologie dell'Informazione (CNR-ISTI) is the organizer of the school on "2D/3D documentation for archaeology". The school aims at providing training and hands-on experience on the tools for producing and managing $2 \mathrm{D}$ and $3 \mathrm{D}$ documentation for archaeological purposes, both for objects and for monuments/sites, including the 
related visualisation tools. The school will provide an introduction to the technologies and tools and a hands-on activity, organizing the students in small groups. Students will bring their own research project, so that they can practise on case studies of direct interest for them.

\subsection{Design of archaeological datasets}

The NeMIS Laboratory of CRN-ISTI develops technologies for modelling access and handling of information, digital library services and services for information retrieval.

NeMIS Lab is organizing the summer school on "Design of archaeological datasets" with the aim to provide students with an introduction to metadata design for archaeological datasets, with perspective content provided as case studies by the students. The school will consist of some introductive lectures followed by hands-on seminars in which the design is developed by the students with the supervision of ISTI experts and then collectively discussed.

\section{FUTURE OPPORTUNITIES}

The three summer schools organized for year 2014 are not the sole opportunities offered by ARIADNE. Until October 2016, ARIADNE will advertise calls for proposals for researchers and professionals to apply for either individual access visits or for summer schools at one of three research centres: PIN, ISTI-CNR and Athena Research Centre.

In particular, opportunities to learn about legacy dataset design and implementation of archaeological datasets and thesauri, conversion of legacy datasets and data formats, CIDOC CRM compliance, definition of metadata schemas, metadata mapping, semantic annotation, and the use of standard languages such as OWL, SKOS will be provided by PIN, ISTI-CNR and the Data Curation Unit of the Athena Research Centre.

Moreover the Cultural and Educational Technology Institute (CETI) will provide scientific assistance and guidance to researchers on the development of scientific datasets: dating techniques for archaeological artefacts (TL and/or OSL), composition, structure and organic residues.

\section{REFERENCES}

ARIADNE http://www.ariadne-infrastructure.eu CIDOC CRM http://www.cidoc-crm.org

PIN, VAST-LAB http://www.vast-lab.org

ISTI-CNR http://www.isti.cnr.it

Athena Research Centre http://www.athena-innovation.gr/en.html 